\title{
Rheumatoid lung disease, pneumothorax, and eosinophilia
}

\author{
A. J. CRISP, R. D. ARMSTRONG, R. GRAHAME, AND J. E. DUSSEK
}

From the Departments of Rheumatology and Thoracic Surgery, Guy's Hospital, London SE1 9RT

SUMmARY Four cases of the triad of rheumatoid lung disease, spontaneous pneumothorax, and peripheral blood eosinophilia are reported. Cavitation of a rheumatoid lung nodule caused the pneumothorax in at least 1 case. The significance of eosinophilia and its value as a marker of extra-articular manifestations of rheumatoid disease are discussed.

The complication of spontaneous pneumothorax secondary to nodular rheumatoid lung disease is rare but occasionally described. ${ }^{1-4}$ Portner and Gracie described 2 patients who also had eosinophilia, ${ }^{5}$ and it has been shown that eosinophilia is commoner in the presence of several extra-articular manifestations of rheumatoid disease. ${ }^{6}$ The 4 cases here described represent further examples of this triad, and it is argued that eosinophilia-even in the presence of mild arthritis-demands a search for extra-articular features of the disease when simple explanations such as chrysotherapy or parasitic infection have been excluded.

\section{Case reports}

\section{CASE 1}

A 42-year-old teacher with no previous occupational exposure to coal or other noxious dust developed a symmetrical mild polyarthritis without subcutaneous nodules affecting wrists, shoulders, and knees. His past medical history included recurrent sinusitis and chest infections in childhood for which he had received external radiotherapy to his throat and chest in Germany at the age of 11 years. A long history of low back pain was attributed to radiologically obvious disc degeneration, and he had suffered an attack of hepatitis 5 years previously. He smoked heavily and suffered mild rhinitis. Both his paternal uncle and grandmother have had rheumatoid arthritis. Five months after the onset of malaise and polyarthritis he developed worsening dyspnoea and nonproductive cough over 3 weeks without pain. A chest radiograph revealed bilateral pneumothoraces larger on the right than the left with small pleural effusions. Other investigations included: haemoglobin $15 \cdot 2 \mathrm{~g} / \mathrm{dl}$; white blood cells $16 \cdot 1 \times 10^{9} / 1$ (9982 neutrophils/ $\mu \mathrm{l} ; 2737$ eosinophils/ $\mu$ l (17\%); 2415 lymphocytes/ $\mu$; 805 monocytes $/ \mu \mathrm{l} ; 161$ basophils $/ \mu \mathrm{l}$ ) (SI conversion: $\mathrm{n} / \mathrm{l}=\mathrm{n} / \mu \mathrm{l}$ $\times 10^{6}$ ); ESR 48; latex test positive; sheep cell agglutination titre 512; antinuclear factor test (ANF) negative; hepatitis $B$ antigen negative. Skeletal radiographs showed only very mild juxta-articular osteoporosis in the hands.

Despite conservative management with underwater sealed drainage the lung failed to expand normally and he underwent thoracotomy (J.E.D.). The right lung was covered by thick inelastic fibrin, and the apex of the right upper lobe was adherent to the mediastinum and chest wall. Multiple nodules were noted along the free edge of the upper lobe, and one was excised. A thorough decortication and parietal pleurectomy were performed. Histology of the nodule showed central necrosis and typical features of a rheumatoid nodule abutting on the lung surface (Fig. 1). Postoperatively the right lung remained expanded, and underwater sealed drainage of the left pleural cavity allowed full re-expansion of the left lung. The patient then remained well for the next 4 months, but returned with 2 weeks' worsening dyspnoea without chest pain and signs of a left pneumothorax. As on the right, the apex of the lung was adherent with a thick, inelastic visceral pleura covering both lobes, and decortication of the nonadherent lung was performed. He developed a right frozen shoulder during his recovery, but since its onset his arthritis has been mild and easily controlled with nonsteroidal anti-inflammatory drugs. His total white cell and differential counts have returned to normal. 


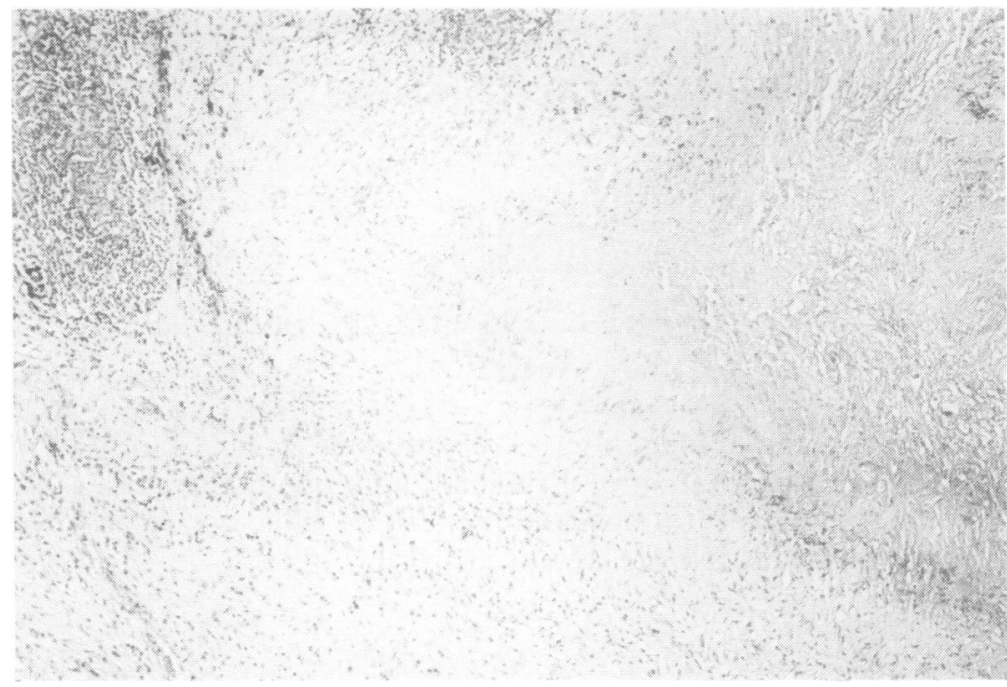

Fig. 1 Edge of a rheumatoid nodule seen on the pleural surface of an open lung surface. (Haematoxylin and eosin; $\times$ 195).

\section{CASE 2}

A 55-year-old man with a 7-year history of seropositive rheumatoid arthritis was noted on $x$-ray to have bilateral small pleural effusions, and some 2 months later he developed a left pneumothorax. No rheumatoid lung nodules were positively identified. An open pleural biopsy was performed, which showed thick granulation tissue with numerous giant cells. Collections of lymphocytes and plasma cells with only scanty neutrophils were also noted, and the pattern was considered to be compatible with, though not diagnostic of, rheumatoid pleural involvement. Despite operative intervention he developed an empyema, which resolved after 18 months. Three months later he developed a left spontaneous pneumothorax. One month after the first pneumothorax, a total white count of $11.3 \times 10^{9} \mathrm{~g} / \mathrm{dl}$ with 1130 eosinophils $/ \mu \mathrm{l}(10 \%)$ was recorded, which later subsided to normal.

\section{CASE 3}

A 65-year-old man with a 27 -year history of seropositive nodular rheumatoid arthritis developed a spontaneous left pneumothorax. Gross joint destruction and deformity, marked muscle wasting, and a peripheral sensorimotor neuropathy were also present. The lung failed to expand with intercostal drainage, and he underwent a pleurectomy (J.E.D.). However, expansion was not maintained, and a second thoracotomy was performed at which all fresh leaking points were sealed. His subsequent course was complicated by an empyema, from which serratia was cultured, and by a large bronchopleural fistula.
Despite continuous suction, intrapleural antiseptics, and appropriate antibiotics he died. During the very early stages of his terminal illness his total white cell count rose to $23.3 \times 10^{9} / 1$ with a $9 \%$ eosinophilia (2097 eosinophils/ $\mu \mathrm{l}$ ).

\section{CASE 4}

A 50-year-old woman with a 15-year history of rheumatoid arthritis had a recurrent left pleural effusion for 10 years treated by intermittent aspiration. She developed a left pyopneumothorax from which Staphylococcus aureus was cultured and was treated successfully with cloxacillin, gentamicin, noxythiolin (Noxyflex) irrigations, and intercostal drainage. Five weeks after the onset of the pyopneumothorax a $12 \%$ eosinophilia was recorded (total white cell count 13.4 $\times 10^{9} / 1 ; 1608$ eosinophils $\left./ \mu \mathrm{l}\right)$. One week later the absolute eosinophil count had risen to $1980 / \mu \mathrm{l}(20 \%$, of a total white cell count of $\left.9.9 \times 10^{9} / 1\right)$. With further clinical improvement the absolute eosinophil count returned to normal $(107 / \mu \mathrm{l})$ (SI conversion: $\mathrm{n} / \mathrm{l}=$ $\left.\mathrm{n} / \mu \mathrm{l} \times 10^{6}\right)$.

\section{Discussion}

Four intrathoracic complications of rheumatoid disease are generally accepted: pleural effusion, ${ }^{7}$ fibrosing alveolitis, ${ }^{8}$ intrapulmonary nodules, ${ }^{4}$ and Caplan's syndrome. ${ }^{9}$ Other lesions reported include acute pulmonary infiltrates, ${ }^{10}$ dry pleurisy and pleural thickening, ${ }^{11}$ and sterile or purulent empyema. ${ }^{12}$ It is of interest that nodules were not obvious on chest radiographs in our first patient and 
the indefinite lesions at both apices (Fig. 2) could easily be explained by the pleural fibrosis obvious at thoracotomy. The fibrosis in turn could reflect either rheumatoid disease per se or possibly the previous course of radiotherapy. Petrie et al. have recently described 4 patients in whom cavitation was adjudged to have occurred in areas of upper lobe fibrosis. ${ }^{13}$ However, as our first case demonstrates, in the presence of fibrosis nodules cannot be excluded by radiography, and only direct vision at thoracotomy can confirm them.

The complication of pneumothorax secondary to nodular rheumatoid lung disease is previously described. It may be significant that both episodes in our first patient were painless, suggesting the gradual erosion of visceral pleura overlying the nodule rather than the usually sudden and painful acute pneumothorax of the young and the emphysematous. The sinister complication of bronchopleural fistula may be more common in conservatively managed cases, ${ }^{4}$ and an active approach with early decortication and pleurectomy if underwater sealed drainage fails may seem well advised.

The association of eosinophilia with these intrathoracic events is of great interest. Portner and Gracie followed up a patient with relative eosinophilia over 6 years of pulmonary nodular activity with pneumothorax. ${ }^{5}$ A $39 \%$ eosinophilia was recorded at the stage of 5 pulmonary nodules, bilateral pleural effusions, and life-threatening bronchopleural fistula. Later, during a prolonged remission from pulmonary involvement but in the presence of subcutaneous nodules, the eosinophil count was followed down to $4 \%$. In their second patient a peripheral blood eosinophilia of $46 \%$ with increased bone marrow counts was recorded at the stage of a single cavitating nodule with pleural effusion. This patient developed a pneumothorax shortly afterwards.
In a severely affected group of patients with rheumatoid disease Winchester et al. found that $40 \%$ of patients had unexplained eosinophil counts of $5 \%$ or more. ${ }^{6}$ Vasculitis, pleuropericarditis, pulmonary fibrosis, and subcutaneous nodules were all commoner in the 'eosinophilic' group. They also noted that certain extra-articular manifestations appeared coincidentally with the development of eosinophilia: nodules, mononeuritis multiplex, peripheral vasculitis, cutaneous ulcers, small ülcerating nodules, pleuropericarditis, episcleritis, and scleromalacia and mesenteric infarction. In $80 \%$ of episodes of eosinophilia a significant new clinical event was recorded. Nodular rheumatoid lung disease, pneumothorax, and eosinophilia may therefore be linked by more than chance.

Gettner et al. have described a patient with a hypereosinophilic syndrome with pulmonary infiltrates who developed bilateral spontaneous pneumothoraces 4 months before the onset of lymphoblastic leukaemia ${ }^{14}$ but the precise role of the eosinophilia remains obscure.

Our present cases support the conclusion that the appearance of eosinophilia in rheumatoid disease may indicate a more aggressive course with extra-articular complications.

We thank Dr. J. M. Heaton for the histological report in case 1 and photograph of the rheumatoid pulmonary nodule; and Dr T. J. Gibson for permission to report the second case which was under his care.

\footnotetext{
References

1 Cudkowicz L, Madoff I M, Abelmann W H. Rheumatoid lung disease. A case report which included respiratory function studies and a lung biopsy, Br J Dis Chest 1961; 55: 35-40.

${ }^{2}$ Hindle W, Yates D A H. Pyopneumothorax complicating rheumatoid lung disease. Ann Rheum Dis 1965; 24: 57-60.
}

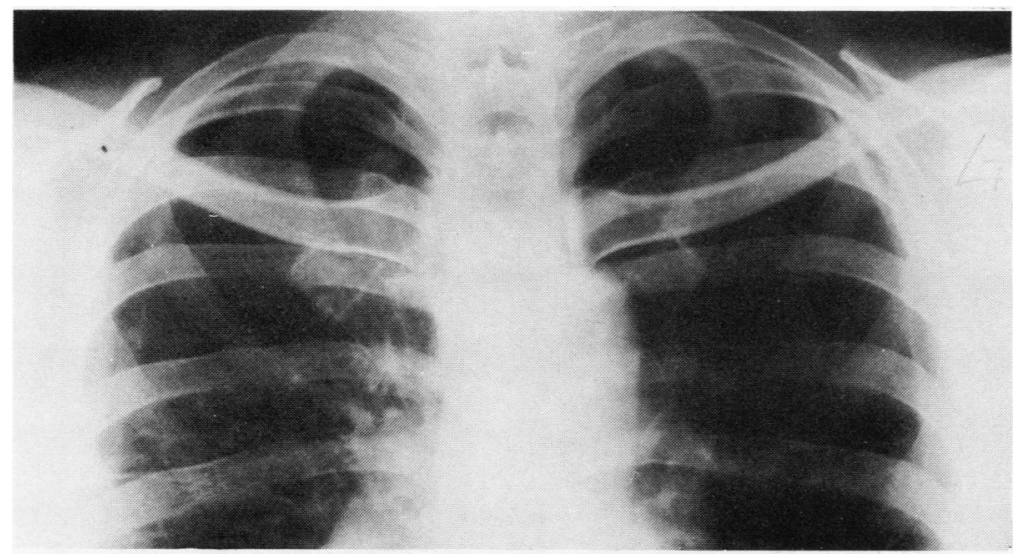

Fig. 2 After resolution of the second pneumothorax in case 1 at least 2 apical lesions are visible. These may represent only fibrosis rather than rheumatoid nodules. 
${ }^{3}$ Davies D. Pyopneumothorax in rheumatoid lung disease. Thorax 1966; 21: 230-5.

4 Rubin E H, Gordon M, Thelmo W L. Nodular pleuro-pulmonary rheumatoid disease. Am J Med 1967; 42: 567-81.

5 Portner M M, Gracie W A. Rheumatoid lung disease with cavitary nodules, pneumothorax and eosinophilia. $N$ Engl J Med 1966; 275: 697-700.

${ }^{6}$ Winchester R J, Litwin S D, Koffler D, Kunkel H G. Observations on the eosinophilia of certain patients with rheumatoid arthritis. Arthritis Rheum 1971; 14: 650-65.

7 Walker W C, Wright V. Pulmonary lesions and rheumatoid arthritis. Medicine 1968; 47: 501-20.

- Scadding J G. The lungs in rheumatoid arthritis. Proc $R$ Soc Med 1969; 62: 227-38.

- Caplan A. Certain unusual radiological appearances of the chest of coal miners suffering from rheumatoid arthritis. Thorax 1953; 8: 29-37.

${ }^{10}$ Beck E R, Hoffbrand B I. Acute lung changes in rheumatoid arthritis. Ann Rheum Dis 1966; 25: 459-62.

11 Gardner D L. The Pathology of Rheumatoid Arthritis. London: Edward Arnold, 1972: 137-43.

12 Dieppe P A. Empyema in rheumatoid arthritis. Ann Rheum Dis 1975; 34: 181-5.

13 Petrie G R, Bloomfield P, Grant I W B, Crompton G K. Upper lobe fibrosis and cavitation in rheumatoid disease. $\mathrm{BrJ}$ Dis Chest 1980; 74: 263-7.

14 Gettner D, Friedman G, Naparstek E, Granot H, Polliack A. Acute lymphoblastic leukaemia. Its occurrence with hypereosinophilic syndrome and bilateral spontaneous pneumothorax. Arch Intern Med 1978; 138: 292-3. 